\title{
Sintering of Soft Magnetic Material under Microwave Magnetic Field
}

\author{
Sadatsugu Takayama, ${ }^{1}$ Jun Fukushima, ${ }^{1}$ Junichi Nishijo, ${ }^{2}$ Midori Saito, ${ }^{2}$ \\ Saburo Sano, ${ }^{3}$ and Motoyasu Sato ${ }^{1}$ \\ ${ }^{1}$ National Institute for Fusion Science, 322-6 Orochi-cho, Toki-shi, Gifu 509-5292, Japan \\ ${ }^{2}$ Institute for Molecular Science, 38 Nishigo-Naka, Myodaiji, Okazaki-shi, Aichi 444-8585, Japan \\ ${ }^{3}$ National Institute of Advanced Industrial Science and Technology, 2266-98 Anagahora, Shimoshidami, Moriyama-ku, \\ Nagoya, Aichi 463-8560, Japan \\ Correspondence should be addressed to Sadatsugu Takayama, takayama.sadatsugu@nifs.ac.jp \\ Received 1 August 2011; Revised 2 January 2012; Accepted 12 January 2012 \\ Academic Editor: Arcady Zhukov
}

Copyright (๑) 2012 Sadatsugu Takayama et al. This is an open access article distributed under the Creative Commons Attribution License, which permits unrestricted use, distribution, and reproduction in any medium, provided the original work is properly cited.

We have developed a simple process for sintering of soft magnetization materials using microwave sintering. The saturated magnetization (Ms) of sintered magnetite was $85.6 \mathrm{emu} / \mathrm{g}$, which was as high as $95 \%$ of magnetite before heating $(90.4 \mathrm{emu} / \mathrm{g})$. On the other hand, the averaged remanence $(\mathrm{Mr})$ and coercivity $(\mathrm{Hc})$ of the magnetite after heating were $0.17 \mathrm{emu} / \mathrm{g}$ and $1.12 \mathrm{Oe}$ under measuring limit of SQUID, respectively. For the sintering process of soft magnetic materials, magnetic fields of microwave have been performed in nitrogen atmosphere. Therefore, a microwave single-mode system operating at a frequency of $2.45 \mathrm{GHz}$ and with a maximum power level of $1.5 \mathrm{~kW}$ was used. We can sinter the good soft magnetic material in microwave magnetic field. The sample shrank to $82 \%$ theoretical density (TD) from $45 \%$ TD of green body. The sintered sample was observed the microstructure by TEM and the crystal size was estimated the approximate average size is $10 \mathrm{~nm}$.

\section{Introduction}

Sintering of magnetite $\left(\mathrm{Fe}_{3} \mathrm{O}_{4}\right)$ with nanocrystals has long been of great interest because of their immense technological applications especially in the magnet, motor, and electric parts. Nanoparticles with superparamagnetic properties have great potential to achieve such desirable properties.

Recently, various methods have been developed to synthesize $\mathrm{Fe}_{3} \mathrm{O}_{4}$ particles in nanometer size range. These methods were the electrochemical synthesis [1], the reactive magnetron sputtering [2], chemical reaction [3], and so on. However, the magnetic properties of magnetite-based nanoparticles or films highly depend upon the synthesis procedure.

Microwave irradiation to materials is a new comer for our civilization with a history of only half century. The temperatures of the surroundings are colder than that of targets, that can easily be imagined by a home microwave oven. It clearly suggests that the energy transfer mechanism in microwave heating is quite different from the traditional heating process. Roy et al. reported sintering of metal powders by microwave in 1999 [4] and decrystallization of ferrite magnetic materials by microwave magnetic field heating in 2002 [5]. Therefore, we have studied the sintering of soft magnetization under microwave heating.

\section{Experimental Setup}

The samples had been heated by magnetic field of microwave. Figure 1 shows a schematic drawing of experimental setup of magnetic field heating of $2.45 \mathrm{GHz}$ microwave. The magnetic or electric fields of microwave can be separated on positions in the single-mode cavity. High-frequency alternated magnetic field was applied to a sample placed on the magnetic field node in the TE103 single-mode cavity with the cross-section of $27.2 \mathrm{~mm} \times 85 \mathrm{~mm}$. The generator, PRJ-1000L, Ewig Co., Ltd., supplied microwave to the cavity at the frequency $2.45 \mathrm{GHz}$. The microwave power 


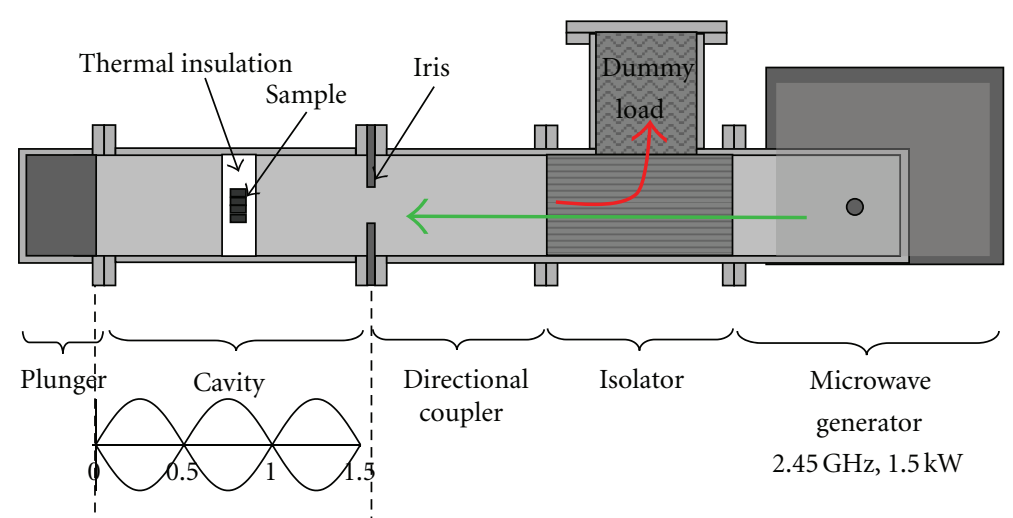

FIGURE 1: The schematic drawing of experimental setup of microwave heating.

varied from 50 to 1500 watts controlling by the DC power supply which consisted of AC-DC inverter. The infrared pyrometer, IGAR12-LO, IMPAC Infrared Co., Ltd., measured the temperature of the sample through the $6 \mathrm{~mm}$ hole drilled through the end plunger of cavity. The waveguide was evacuated to $10^{-4} \mathrm{~Pa}$ by turbo molecular pump with $100 \mathrm{~L} / \mathrm{s}$ pumping speed and changed to nitrogen gas in the cavity. During the heating, nitrogen gas was flowing in cavity.

The samples were prepared by uniaxial pressing of magnetite powder (FEO07PB) with the purity of 99 weight $\%$ from Kojundo Chemical Laboratory Co., Ltd., Japan. The sample density was up to $45 \%$ of the theoretical density (TD) by geometrical method. The grain size of the magnetite was less than 1 micrometer. The size of the sample was $8 \mathrm{~mm}$ diameter and $4 \mathrm{~mm}$ thick pellet of $0.5(\mathrm{~g})$ that was small enough not to disturb the criterion for fundamental resonance in the cavity. The four samples were put in the magnetic field maxima in the cavity supported by thermal insulator made of a lightweight alumina silica fiber board.

\section{Experimental}

The graphs in Figure 2 show a typical progress of process temperatures measured during microwave heating of magnetite samples. The microwave power was controlled manually. The microwave power shows remainder of input power and reflex power. For sintering experiments, the magnetite powder compacts were heated to the sintering temperature of $1000^{\circ} \mathrm{C}$ for $10 \mathrm{~min}$ followed by $70 \mathrm{~min}$ soak time. In initial step heating during the first $30 \mathrm{~min}$, the sample temperature was dramatically changing. Thereafter, the sample temperature was settling and microwave power was lower as 270 watt.

Figure 3 shows a magnetite sample sintered by using $\mathrm{H}$ filed of microwaves at $1000^{\circ} \mathrm{C}$. The sample shrank to $6.2 \mathrm{~mm}$ diameter from $8 \mathrm{~mm}$ diameter for sintering. The sintered sample was found to be oxidized on the only surface. The linear shrinkage of the sample was $17 \%$ and no remarkable change in the shape was observed. The sample shrank to $82 \%$ theoretical density (TD) from 45\% TD of green body.

Figure 4 shows the $\mathrm{X}$-ray diffraction profile of the magnetite after heating at $1000^{\circ} \mathrm{C}$. It shows Bragg reflections of

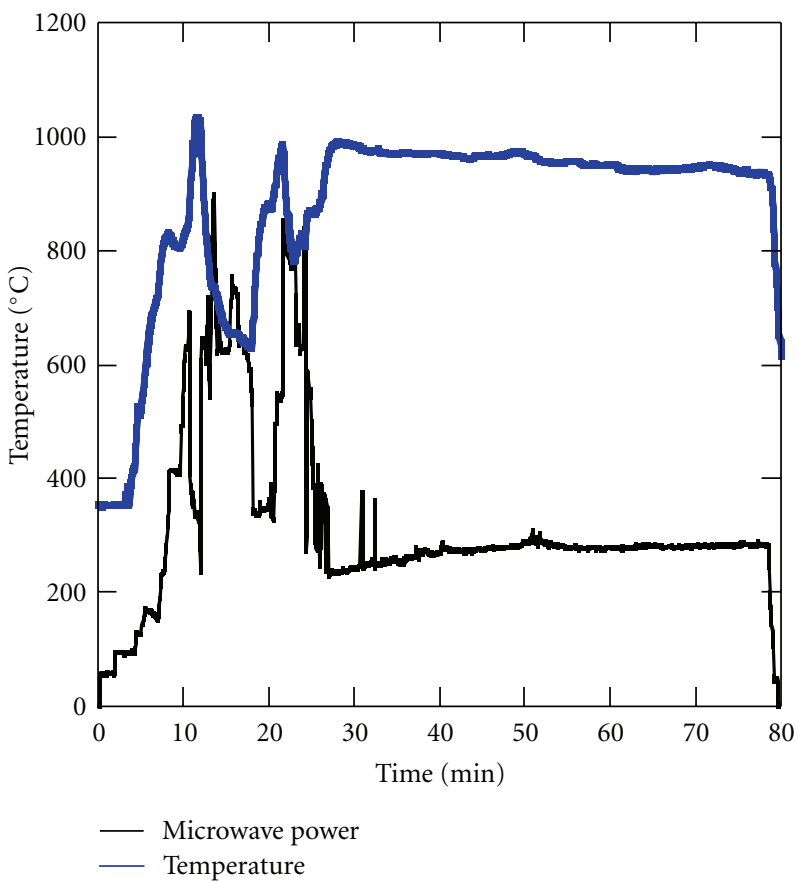

FIGURE 2: The typical progress of process temperature measured during microwave heating.

both magnetite and hematite. The surface of sample was oxidized because the nitrogen gas was flowing in cavity during heating.

The high-resolution transmission electron microscope (TEM; a JEOL JEM-3200) observed the images of the original magnetite powder before heating and those excited in the selected magnetic field. The original crystal can see the wellordered lattice patterns over the whole crystal; therefore the original crystal has flat and homogeneous surfaces. Figure 5 shows TEM image and selected area electron diffraction pattern. The sample heated in the magnetic field exhibits the presence of randomly oriented nano-crystal. The crystal sizes of this sample are of approximate average size $10 \mathrm{~nm}$. The randomness of the lattice orientation indicates that the particle-particle magnetic interaction is negligible in 


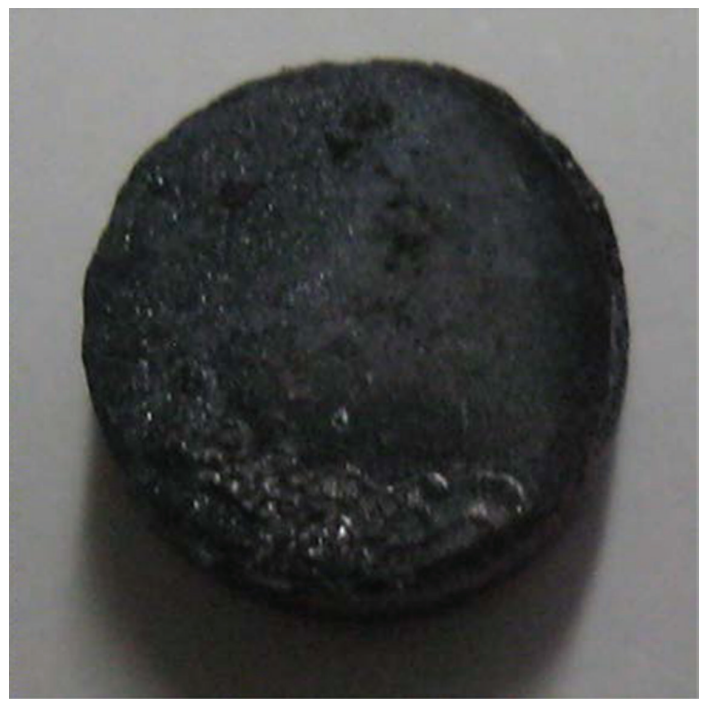

FIGURE 3: The picture of magnetite sintered by using magnetic field of microwave at $1000^{\circ} \mathrm{C}$.

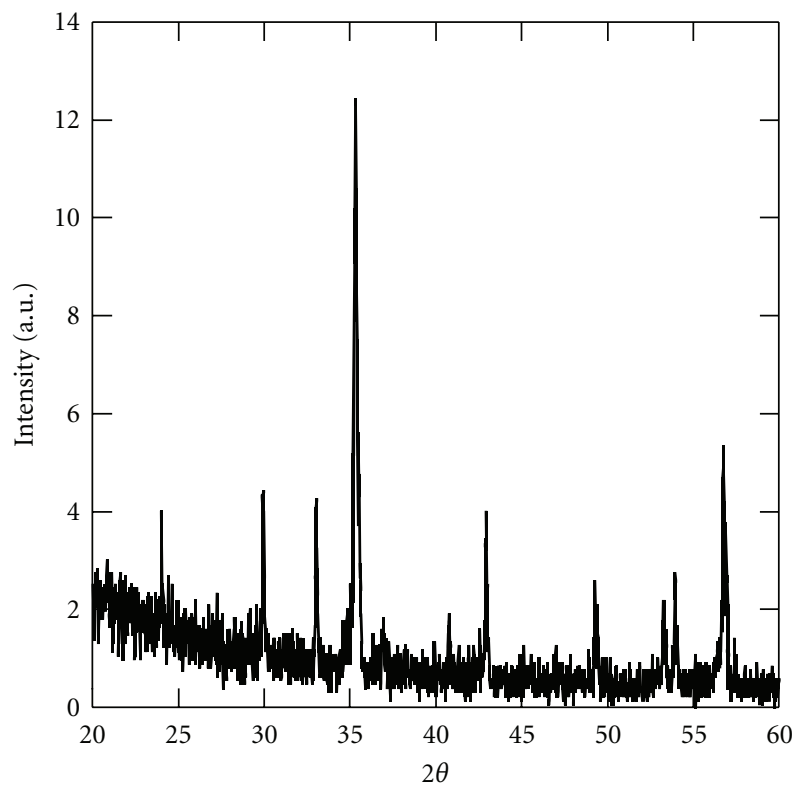

Figure 4: X-ray diffraction of samples heated by magnetic field at $1000^{\circ} \mathrm{C}$.

the heating cooling process. If each magnetic domain should be highly excited by application of the oscillating magnetic field, cohesive rotation of the domains can be induced synchronously with the oscillating field. It is very interesting that the temperature increase in the sample is accelerated at a temperature close to the Curie point and never rises above $1000^{\circ} \mathrm{C}$ but it is well below the melting point of $1535^{\circ} \mathrm{C}$.

The macroscopic magnetizations of samples were measured by SQUID without zero correction (Quantum Design MPMS-XL7). Figure 6 shows the hysteresis loop of magnetite at $300 \mathrm{~K}$. As can be seen in Figure 6, the saturated magnetization (Ms) of sintered magnetite was $85.6 \mathrm{emu} / \mathrm{g}$, which was as high as $95 \%$ of magnetite before heating ( $90.4 \mathrm{emu} / \mathrm{g})$.

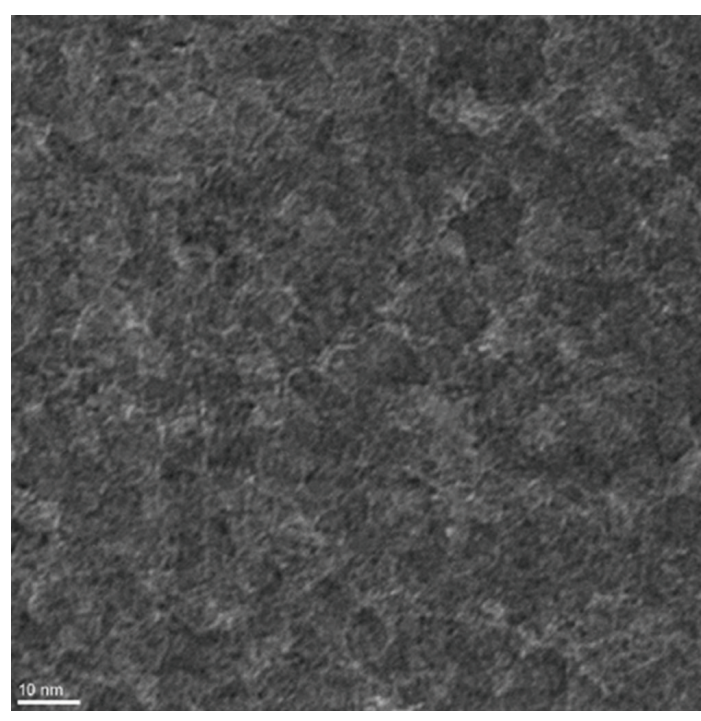

(a)

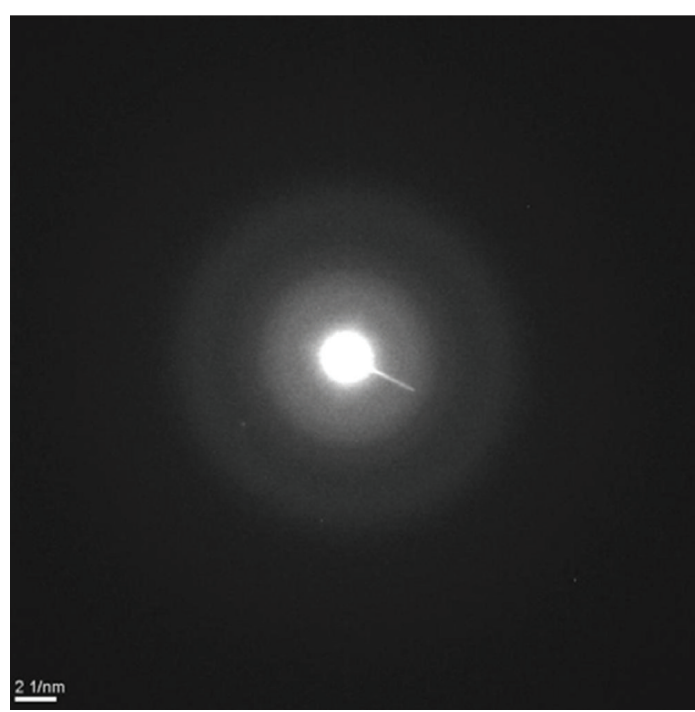

(b)

FIGURE 5: TEM image of magnetite sintered by using magnetic field of microwave at $1000^{\circ} \mathrm{C}$.

The averaged remanence ( $\mathrm{Mr}$ ) and coercivity ( $\mathrm{Hc}$ ) of the magnetite before heating were $11.74 \mathrm{emu} / \mathrm{g}$ and $108.9 \mathrm{Oe}$, respectively. On the other hand, the averaged remanence $(\mathrm{Mr})$ and coercivity $(\mathrm{Hc})$ of the magnetite after heating were $0.17 \mathrm{emu} / \mathrm{g}$ and $1.12 \mathrm{Oe}$ under measuring limit of SQUID, respectively. Their $\mathrm{Mr}$ value and $\mathrm{Hc}$ value of sintered sample became two digits smaller than raw sample for heating by magnetic field of microwave.

\section{Conclusion}

We have developed a simple process for sintering of soft magnetization materials. For the sintering process of soft magnetic materials, magnetic fields of microwave have been performed in nitrogen atmosphere. Therefore, a microwave 


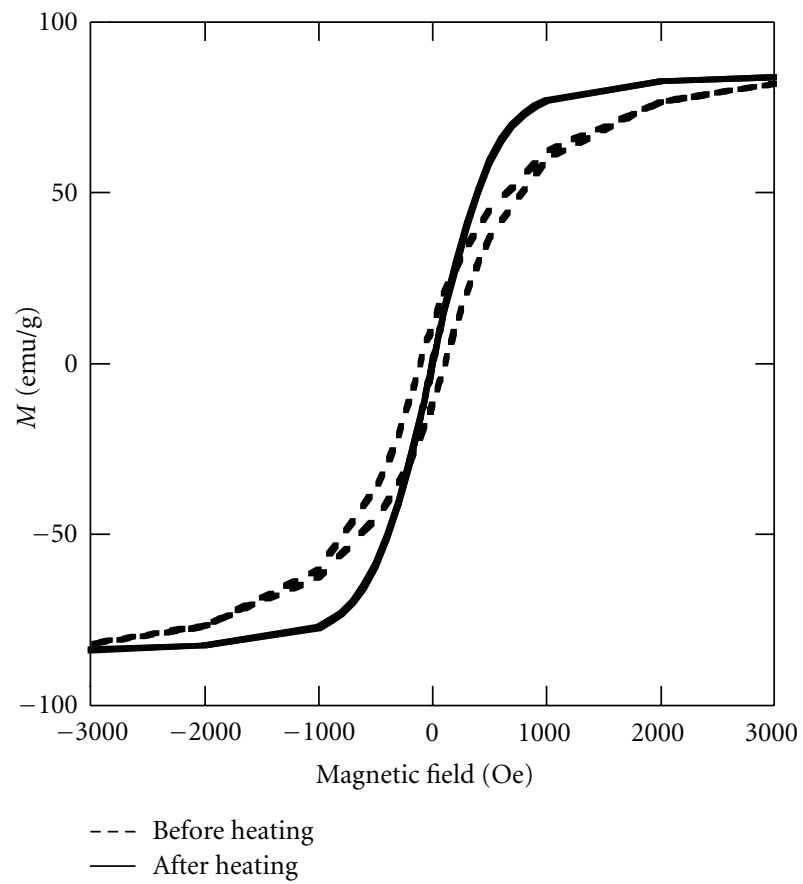

(a)

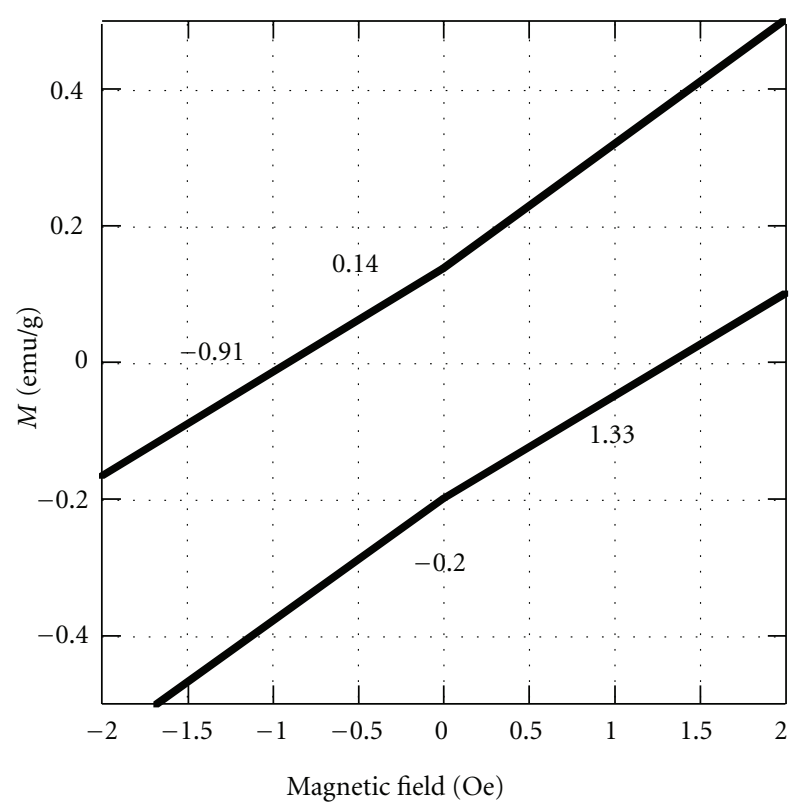

(b)

Figure 6: (a) The hysteresis loop of the magnetite sintered by using magnetic field of microwave at $1000^{\circ} \mathrm{C}$. (b) The $\mathrm{Mr}$ and $\mathrm{Hc}$ of the magnetite sintered by using magnetic field of microwave at $1000^{\circ} \mathrm{C}$.
Using microwave sintering, the Ms of sintered magnetite was $85.6 \mathrm{emu} / \mathrm{g}$, which was as high as $95 \%$ of raw magnetite with $90.4 \mathrm{emu} / \mathrm{g}$. On the other hand, the $\mathrm{Mr}$ and $\mathrm{Hc}$ of the magnetite after heating were $0.17 \mathrm{emu} / \mathrm{g}$ and $1.12 \mathrm{Oe}$ under measuring limit of SQUID, respectively.

From TEM and SQUID data, it is suggested that the sintered magnetite under magnetic field of microwave has super-ferrimagnetism.

We expect that it is used in transformer or electromagnetic cores. The macroscopic magnetization of sintered body using microwave can easily reverse direction without dissipating much energy, that is, hysteresis losses. Because of their comparatively low losses, they are extensively used in the cores of transformers and electromagnetic cores in applications such as car industrials.

\section{References}

[1] L. Cabrera, S. Gutierrez, N. Menendez, M. P. Morales, and P. Herrasti, "Magnetite nanoparticles: electrochemical synthesis and characterization," Electrochimica Acta, vol. 53, no. 8, pp. 3436-3441, 2008.

[2] L. Pan, G. Zhang, C. Fan et al., "Fabrication and characterization of $\mathrm{Fe}_{3} \mathrm{O}_{4}$ thin films deposited by reactive magnetron sputtering," Thin Solid Films, vol. 473, no. 1, pp. 63-67, 2005.

[3] H. K. Lee, S. H. Do, S. H. Kong et al., "The role of magnetite nano particle (MNP) to oxidize nitrobenzene using heterogeneous fenton reaction," in Proceedings of the World Congress on Engineering and Computer Science (WCECS '10), vol. 2, 2010.

[4] R. Roy, D. Agrawal, J. Cheng, and S. Gedevanlshvili, "Full sintering of powdered-metal bodies in a microwave field," Nature, vol. 399, no. 6737, pp. 668-670, 1999.

[5] R. Roy, R. Peelamedu, L. Hurtt, J. Cheng, and D. Agrawal, "Definitive experimental evidence for Microwave Effects: radically new effects of separated $\mathrm{E}$ and $\mathrm{H}$ fields, such as decrystallization of oxides in seconds," Materials Research Innovations, vol. 6, no. 3, pp. 128-140, 2002. single-mode system operating at a frequency of $2.45 \mathrm{GHz}$ and with a maximum power level of $1.5 \mathrm{~kW}$ was used. We can sinter the good soft magnetic material in microwave magnetic field. The sample shrank to $82 \%$ theoretical density (TD) from $45 \% \mathrm{TD}$ of green body. The sintered sample was observed the microstructure by TEM and the crystal size was estimated the approximate average size is $10 \mathrm{~nm}$. 

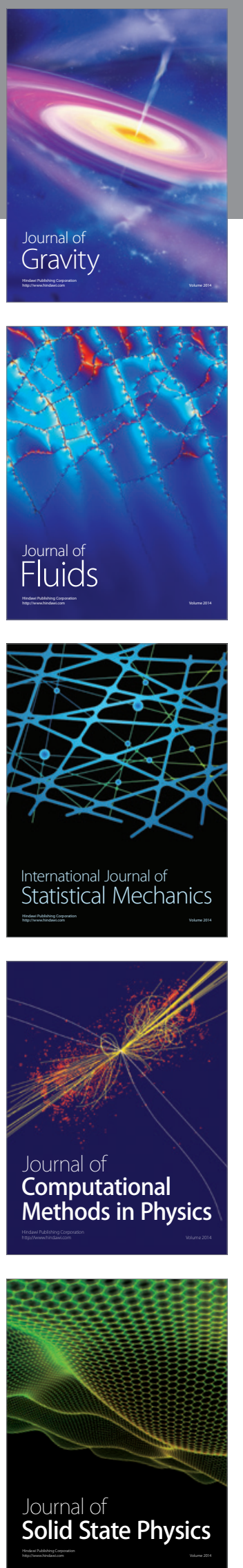

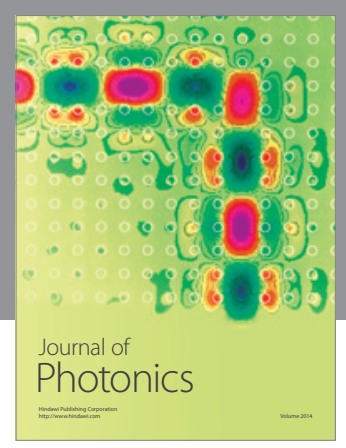

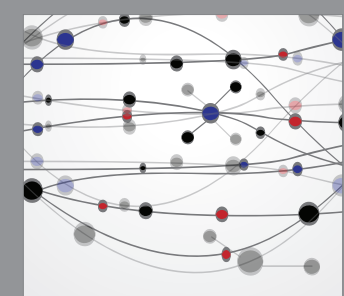

The Scientific World Journal
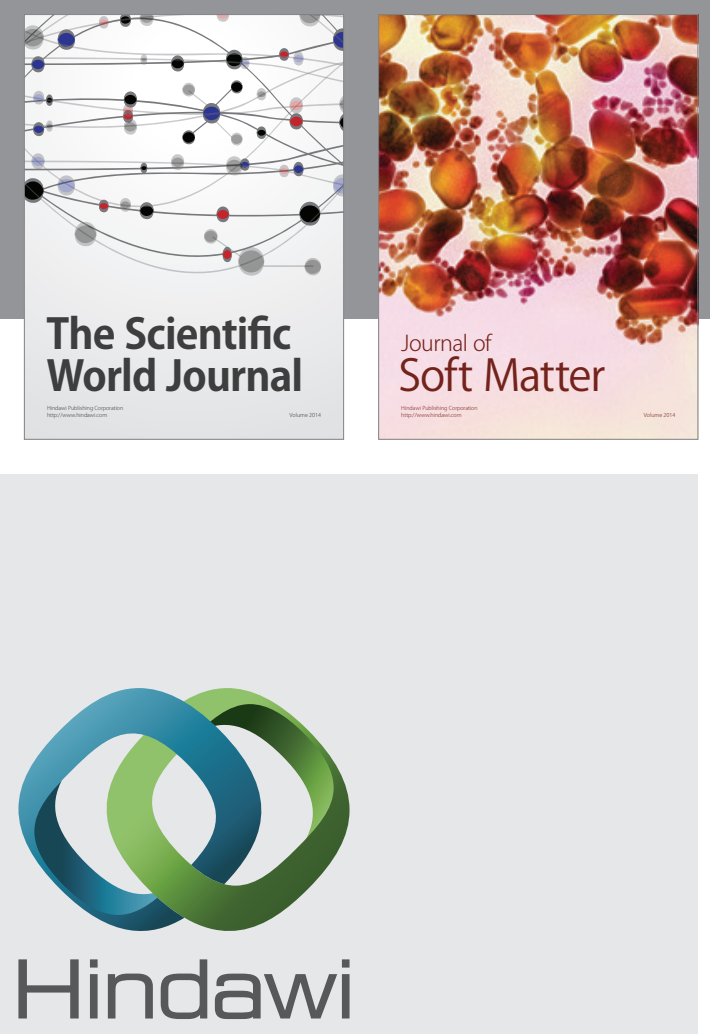

Submit your manuscripts at

http://www.hindawi.com
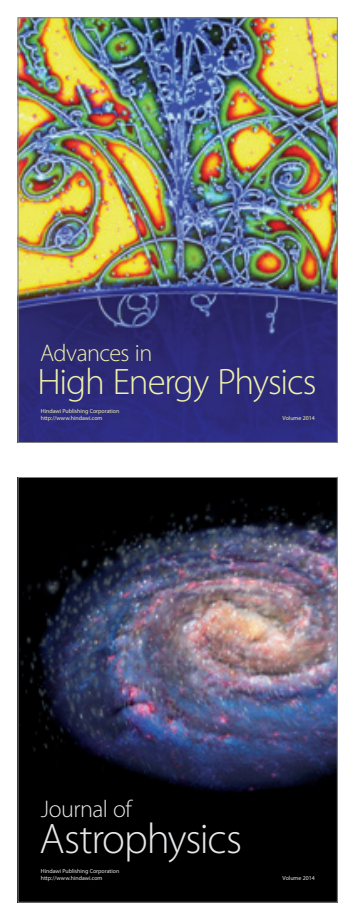
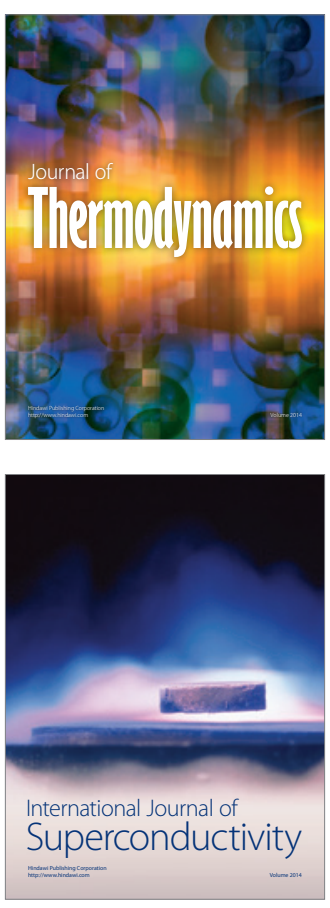
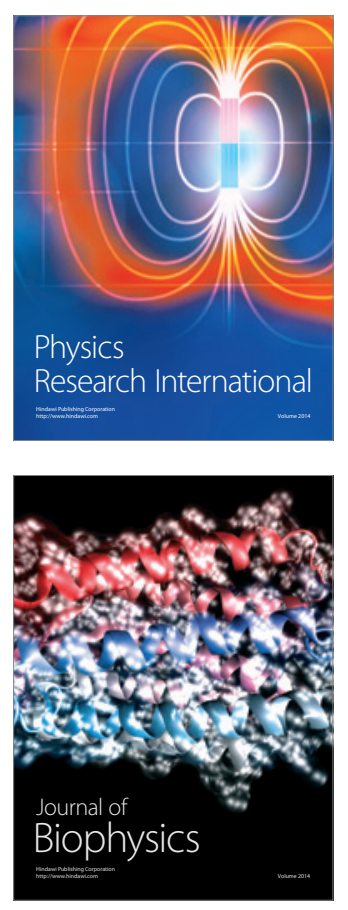
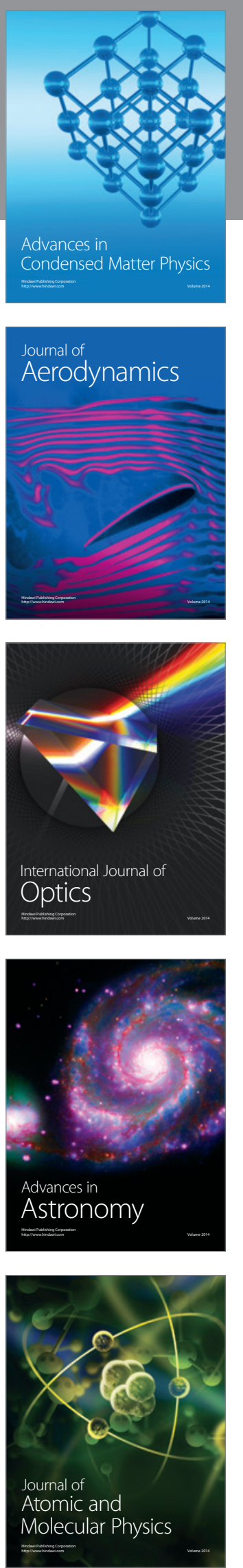